\section{Metabolomics: How it can be applied in Infectious Medicine?}

Bioinformatics becomes a very useful scientific technology at present. It can be applied in several purposes including to medicine. In medicine, the well-known genomics and proteomics have been widely applied in diagnostic and therapeutic researches [1]. However, the new generation of "omics" such as metabolomics is limited mentioned. In fact, metabolomics can be very useful in clarification and prediction of the question on "metabolome". Vinayavekhin et al. noted that "metabolomics offers unique insights into small molecule regulation and signaling in biology [2]". The application in infectious medicine is an actual challenge. Few reports have been published for a few years. Peng et al. noted that "the reprogramming metabolomics approach can be used to clarify metabolic mechanisms of responding to changed internal and external environmental factors and to establish a framework to develop targeted tools for dealing with the changes such as controlling and/or preventing infection with pathogens and enhancing host immunity against pathogens [3].” To understand the disease, several metabolites including "amino acids, organic acids, carbohydrates, nucleosides, lipids, fatty acids, and derivatives" can be traced [4]. However, the big present obstacle is the lack for complete metabolic profile in several diseases. Identification of such profile, database setting and development of new in silico metabolomics tool can be the next step for archiving the success in metabolomics application in infectious medicine.

\section{References}

1. Robbins RJ (1996) Bioinformatics: essential infrastructure for global biology J Comput Biol 3: 465-478.

\section{Journal of}

Metabolomics \& Systems Biology

\section{Viroj Wiwanitkit ${ }^{1,2 *}$}

${ }^{1}$ Public Health Curriculum, Surin Rajabhat University, Surin 33000, Thailand

${ }^{2}$ Dr. D.Y. Patil Medical University, Pune, India

*Address for Correspondence

Viroj Wiwanitkit, Public Health Curriculum, Surin Rajabhat University,

Surin 33000, Thailand, E-mail: wviroj@yahoo.com

Submission: 02 October 2015

Accepted: 12 October 2015

Published: 16 October 2015

Copyright: (c) 2015 Wiwanitkit V. This is an open access article distributed under the Creative Commons Attribution License, which permits unrestricted use, distribution, and reproduction in any medium, provided the original work is properly cited.

Reviewed \& Approved by: Dr. Yi-Lei Zhao, School of Life Sciences and Biotechnology, Shanghai Jiao Tong University, China

2. Vinayavekhin N, Homan EA, Saghatelian A (2010) Exploring disease through metabolomics. ACS Chem Biol 15: 91-103.

3. Peng B, Li H, Peng XX (2015) Functional metabolomics: from biomarker discovery to metabolome reprogramming. Protein Cell 6: 628-637.

4. Canuto GA, da Cruz PL, Faccio AT, Klassen A, Tavares MF (2015) Neglected diseases prioritized in Brazil under the perspective of metabolomics: A review. Electrophoresis [Epub ahead of print] 